\title{
Papel dos lipídeos da dieta na nefropatia diabética
}

\author{
Role of dietary lipids in diabetic nephropathy
}

Jussara C. de Almeida', Vanessa D. de Mello', Luis H. Canani', Jorge L. Gross', Mirela J. de Azevedo'

'Serviço de Endocrinologia, Hospital de Clínicas de Porto Alegre (HCPA), Universidade Federal do Rio Grande do Sul (UFRGS), Porto Alegre, RS, Brasil

Correspondência para:

Mirela J. de Azevedo Serviço de Endocrinologia do HCPA Rua Ramiro Barcelos, 2.350, prédio 12 , 4 andar 90035-003 - Porto Alegre, RS, Brasil mirelaazevedo@terra.com.br

Recebido em 27/Mar/2008 Aceito em 19/Nov/2008

\section{RESUMO}

O objetivo do presente manuscrito foi revisar o possível papel dos lipídeos dietéticos na nefropatia diabética (ND), considerando as alterações do perfil lipídico associadas e a interação entre aspectos dietéticos e genéticos. Os lipídeos dietéticos podem ter um papel importante no desenvolvimento e na progressão da ND. A composição das gorduras da dieta tem sido associada com a ND, particularmente à microalbuminúria e às anormalidades lipídicas e de função endotelial. Entretanto, ainda não está comprovado o benefício da modificação da ingestão de gorduras em pacientes com ND, em especial sobre desfechos definitivos, como incidência e progressão da ND, insuficiência renal e morte. Além disso, a resposta do perfil lipídico à ingestão de gorduras pode ser influenciada por fatores genéticos. A identificação de polimorfismos genéticos específicos associados a essa interação poderá permitir a individualização de estratégias nutricionais na ND. Arq Bras Endocrinol Metab. 2009;53(5):634-45.

Descritores

Nefropatias diabéticas; albuminúria; lipídeos na dieta; ácidos graxos; lipoproteínas; dislipidemia

\section{ABSTRACT}

The aim of the present study was to review the possible role of dietary lipids in diabetic nephropathy (DN), taking into account associated abnormalities of serum lipids and interaction of dietary and genetic aspects. Dietary lipids may have an important role in the development and progression of DN. The fat diet composition has been associated with DN, particularly with microalbuminuria, serum lipids abnormalities, and endothelial function. However, the beneficial effect of fat intake modification for these patients is not fully established, especially regarding hard outcomes, such as DN incidence and progression, kidney failure, and death. Moreover, genetic factors may influence the response of serum lipids to fat intake. The identification of specific genetic polymorphisms associated with this interaction could allow adoption of individual nutritional strategies in DN. Arq Bras Endocrinol Metab. 2009;53(5):634-45.

Keywords

Diabetic nephropathies; albuminuria; dietary fats; fatty acids; lipoproteins; dyslipidemia

\section{INTRODUÇÃO}

A neienticin nefropatia diabética (ND) acomete até $40 \%$ dos pacientes com diabetes melito (DM) e, além de estar associada a uma mortalidade cardiovascular aumentada, é considerada a principal causa de insuficiência renal crônica naqueles pacientes que ingressam em programa de tratamento para a substituição renal (1).

A ND é definida pelo aumento da excreção urinária de albumina na ausência de outras doenças renais e tem sido classificada em estágios. O estágio denominado de microalbuminúria, ou de nefropatia incipiente, caracte- riza-se por valores de excreção urinária de albumina de 30 a $299 \mathrm{mg} / 24$ horas. No estágio de macroalbuminúria, ou nefropatia clínica, os valores correspondentes de excreção urinária de albumina são $\geq 300 \mathrm{mg} / 24$ horas. Já no estágio de microalbuminúria, ocorre aumento de mortalidade, em especial cardiovascular, e um risco aumentado de progressão para insuficiência renal (1).

Para o diagnóstico da ND, a American Diabetes Association recomenda que seja também estimada a taxa de filtração glomerular (TFG), independente dos valores de excreção urinária de albumina (2). Alguns 
pacientes com DM podem apresentar diminuição da TFG na presença de valores normais de excreção urinária de albumina. Esses pacientes normoalbuminúricos e com TFG $<60 \mathrm{~mL} / \mathrm{min}$ apresentam hipertrigliceridemia e síndrome metabólica com mais frequência quando comparados com aqueles com valores superiores de TFG (3). Deve ser também lembrado que valores elevados de excreção urinária de albumina em pessoas com diabetes melito tipo 2 (DMT2) podem também estar relacionados à presença de hipertensão arterial, frequentemente observada nesses pacientes.

A hiperglicemia e o aumento dos níveis de pressão arterial, associados à predisposição genética, são os principais fatores de risco para o desenvolvimento da ND. A obtenção de um controle glicêmico próximo à normalidade, o tratamento rigoroso da hipertensão arterial, o uso de drogas bloqueadoras do sistema renina-angiotensina-aldosterona e, possivelmente, o tratamento da dislipidemia têm sido medidas utilizadas para a prevenção e desaceleração da progressão da ND (1). No entanto, ainda há um número considerável de pacientes que desenvolvem $\mathrm{ND}$, e sua progressão não é completamente evitada com os tratamentos disponíveis atualmente. Portanto, é necessário que outros fatores associados à $\mathrm{ND}$, como o tipo de dieta, sejam mais bem estudados para otimizar a prevenção e o tratamento dessa complicação crônica.

No final da década de 1990, estudos observacionais em pacientes com DM sugeriram que a quantidade (4) ou o tipo (5) de proteína estavam associados à microalbuminúria. Também o conteúdo de gordura (6-10) consumido na dieta usual parece ser um fator de risco para o desenvolvimento e progressão da microalbuminúria. De fato, em pacientes com DMT2, já foi demonstrado que quando é utilizada carne branca em substituição à vermelha, a consequente modificação do conteúdo de ácidos graxos poli-insaturados (AGPIs) e saturados (AGSs) da dieta reduz a excreção urinária de albumina e melhora o perfil lipídico (11-12). É improvável que tal efeito seja devido a modificações nos aminoácidos plasmáticos, pois esses não se modificam após três semanas de dieta à base de carne de galinha (13). Postula-se que esses efeitos ocorram por causa da alteração dos ácidos graxos (AGs) séricos ou, ainda, pela absorção intestinal modificada de AGs dietéticos. Deve ser lembrado, entretanto, que as alterações nos lipídeos séricos que influenciam o curso clínico da ND podem ocorrer independente da dieta (14).

A dislipidemia está presente em pacientes com ND desde a fase de microalbuminúria. É possível que essas alterações nos AGs séricos estejam associadas às alterações inflamatórias e de função endotelial, como já descrito em pacientes sem DM (15). Recentemente, em pacientes com DMT2, os AGSs séricos se correlacionaram com os valores de endotelina-1 (16). Reforça essa observação a associação positiva entre excreção urinária de albumina e endotelina-1 em pacientes com DMT2 (17). As anormalidades dos AGs séricos podem contribuir para a elevada mortalidade cardiovascular observada em pacientes com DM microalbuminúricos. Deve ser ainda considerada a possibilidade de interação entre fatores ambientais dietéticos e genéticos na promoção de condições favoráveis para o desenvolvimento da ND.

\section{DISLIPIDEMIA E NEFROPATIA DIABÉTICA}

A associação entre um perfil lipídico aterogênico e a excreção urinária de albumina tem sido observada em pacientes com diabetes melito tipo l (DMTl) (18) e DMT2 $(14,19)$. As associações positivas dos lipídeos séricos com a ND foram descritas em relação aos valores de colesterol total, colesterol LDL (low-density lipoprotein), triglicerídeos, VLDL (very low-density lipoprotein) e apolipoproteína $\mathrm{B}$, além de alterações no tamanho das partículas de LDL (19). AGs séricos foram também associados à microalbuminúria no DM. Pacientes com DMT2 e microalbuminúria apresentaram um percentual sérico menor de AGPIs e maior de AGSs na fração triglicerídeo, quando comparados aos pacientes normoalbuminúricos (14). É possível que o tipo de anormalidade lipídica seja distinto em diferentes fases da ND. Em pacientes com DMT1, a progressão da ND foi associada com o colesterol não esterificado (forma livre) da LDL nos pacientes normoalbuminúricos; com os triglicerídeos das VLDL e IDL (intermediary-density lipoprotein) nos microalbuminúricos; e com o menor tamanho das partículas de LDL nos pacientes macroalbuminúricos (18).

É provável que as alterações lipídicas precedam o desenvolvimento da micro ou macroalbuminúria - e não o contrário - embora esse tópico não esteja completamente esclarecido. Em um estudo no qual 133 pacientes com DMT2 foram acompanhados por um período de cinco anos, a presença de microalbuminúria foi um fator de risco para desenvolvimento de hipertrigliceridemia e valores diminuídos de HDL (bigh-density lipoprotein) (19). Entretanto, em fases mais avançadas da ND, os valores elevados de colesterol sérico parecem ser determinantes para a perda de função renal em pacientes com DM (20). 
Um dos prováveis mecanismos pelo qual a dislipidemia contribui para o desenvolvimento e progressão da ND pode estar relacionado ao efeito nefrotóxico dos lipídeos séricos. O aumento da permeabilidade de macromoléculas no mesângio, incluindo lipídeos, levaria à progressão da lesão glomerular inicial para glomerulosclerose. Na ND, outro aspecto interessante em relação ao mecanismo de ação dos lipídeos, em especial o colesterol, é a semelhança histológica e imunoistoquímica entre a lesão glomerular progressiva e o desenvolvimento de estrias gordurosas no processo de aterosclerose. É possível que a patogênese de ambos os processos seja compartilhada (21).

A resistência à insulina, presente, de maneira geral, nos pacientes com DMT2, está também entre os prováveis mecanismos relacionados às alterações lipídicas séricas em pacientes com ND. A redução do efeito da insulina em situações de hiperglicemia favorece a mobilização dos AGs livres do tecido adiposo que, por sua vez, estimula a síntese hepática de apolipoproteína B, principal componente proteico das VLDL, com consequente aumento da produção dessas lipoproteínas que são ricas em triglicerídeos (22). Além disso, a resistência à insulina pode inibir a atividade da enzima lipase lipoproteica (LLP), responsável pela conversão de lipoproteínas ricas em triglicerídeos em AGs livres (23). Como consequência, os valores de colesterol HDL (22) e a depuração de VLDL ficam reduzidos (23).

Tais achados sugerem que as anormalidades lipídicas observadas em pacientes com ND precedam o desenvolvimento da própria ND, em especial em sua fase inicial. Os possíveis mecanismos relacionados à dislipidemia na ND incluem a resistência à insulina e a redução da atividade da enzima LLP. As anormalidades lipídicas resultantes teriam efeito nefrotóxico contribuindo para a patogênese da ND.

\section{EFEITOS DOS LIPÍDEOS DA DIETA SOBRE 0 PERFIL LIPÍDICO SÉRICO}

Os efeitos dos lipídeos da dieta sobre o perfil lipídico sérico são atribuídos especialmente aos AGs, de acordo com seus graus de saturação. Esses efeitos são distintos e independem da presença da DM. Na tabela 1 estão resumidas as principais gorduras da dieta, suas respectivas fontes alimentares e seu impacto sobre os lipídeos séricos, além dos componentes inflamatórios e de coagulação. O colesterol, os AGSs e os AGs insaturados trans-isômeros provenientes da dieta desempenham papel importante nas anormalidades lipídicas, principalmente na elevação de colesterol total e LDL séricos. Os AGMIs, por sua vez, têm um efeito hipocolesterolêmico ao substituírem os AGSs da dieta. A ingestão de AGPIs, em especial os AGs $\omega$-3, além de reduzir os valores de colesterol e triglicerídeos séricos, promove efeitos benéficos nos processos inflamatórios (25), assim como um efeito antitrombogênico (28).

A redução da resistência à insulina e da atividade inflamatória está entre os mecanismos benéficos da modificação do perfil lipídico causada pela quantidade e qualidade de gordura da dieta. Os AGPIs de cadeia muito longa, principalmente o AG docosa-hexaenoico (DHA), diminuem a expressão das moléculas de adesão endotelial, assim como reduzem as interações entre as células do sistema imunológico e as células do endotélio (29). Também já foi demonstrado que a suplementação oral de eicosapentaenoico (EPA) e DHA diminuiu a produção de citoquinas pró-inflamatórias pelas células mononucleares periféricas (30).

Uma maior proporção de AGs insaturados de cadeia longa e uma menor proporção de AGSs na dieta estão associadas à melhora da ação da insulina (31). Os mecanismos associados aos efeitos dos AGs sobre a resistência à insulina são possivelmente relacionados à composição de AGs nas membranas celulares. Na membrana, os AGs influenciariam a sensibilidade à ação da insulina por meio de alteração na afinidade desta com seu receptor, na permeabilidade da membrana à insulina e nos processos de sinalização celular (31). Em pacientes obesos com resistência à insulina, os AGSs apresentaram associação positiva com marcadores inflamatórios séricos (interleucina-6, proteína $\mathrm{C}$ reativa), enquanto para os AGPIs a associação foi negativa (15). Além disso, o papel benéfico dos AGPIs de cadeia muito longa tem sido confirmado em revisões sistemáticas (32-34). A suplementação de EPA e DHA, AGPIs de cadeia muito longa, foi capaz de reduzir a mortalidade geral e cardiovascular (32). Em pacientes com DMT2, a suplementação de EPA e DHA ( 4,3 g/dia) por cerca de nove semanas reduziu em cerca de $25 \%$ os triglicerídeos séricos e em 38\% o VLDL, e aumentou em $25 \%$ o fator VII de coagulação quando comparado com placebo $(33,34)$.

A adoção de uma dieta rica em AGMIs, semelhante à de países mediterrâneos, é uma estratégia primária para a redução de mortalidade por todas as causas (35) e de eventos cardiovasculares (36). Na dieta mediterrânea, há um consumo abundante de vegetais, óleo de 


\begin{tabular}{|c|c|c|}
\hline Gordura da dieta & Fonte alimentar (24) & Impacto no organismo (25) \\
\hline Colesterol dietético & $\begin{array}{l}\text { Produtos de origem animal: vísceras (fígado, coração, cérebro), } \\
\text { manteiga, ovos (gema), tortas com creme ou queijo }\end{array}$ & $\uparrow$ Colesterol total, $\uparrow$ LDL, $\uparrow$ HDL séricos \\
\hline \multicolumn{3}{|c|}{ AG saturados (AGSs) - efeito aterogênico em ordem decrescente } \\
\hline \multirow[t]{2}{*}{$\begin{array}{l}\text { AG palmítico (C16) } \\
\text { AG mirístico (C14) } \\
\text { AG láurico (C12) }\end{array}$} & $\begin{array}{l}\text { Produtos de origem animal: lácteos integrais, carnes de gado } \\
\text { gordas (costelas, cupim, fraldinha, charque, picanha), toucinho } \\
\text { e rabo de porco, ovelha }\end{array}$ & $\begin{array}{l}\uparrow \text { Colesterol total ( } \downarrow \text { da atividade do receptor hepático de LDL } \\
\text { leva a } \downarrow \text { da depuração do LDL e } \uparrow \text { do colesterol sérico) }\end{array}$ \\
\hline & $\begin{array}{l}\text { Produtos de origem vegetal: óleo de coco, babaçu, dendê, } \\
\text { amendoim }\end{array}$ & $\begin{array}{l}\text { Efeito maior do que o efeito resultante da ingestão do próprio } \\
\text { colesterol }\end{array}$ \\
\hline$A G$ com $\leq 10$ carbonos & Óleo de babaçu e coco (mas possuem AG palmítico também) & Neutro em relação aos lipídeos séricos \\
\hline AG esteárico (C18) & Chocolates ${ }^{*}$, carne de cabra & $\begin{array}{l}\uparrow \text { Colesterol total de pouca magnitude, possivelmente pela } \\
\text { rápida conversão a ácido oleico (C18:1 } \omega 9 \text { ) no organismo } \\
\text { \#Efeito final dependente do conteúdo de compostos fenólicos } \\
\text { do cacau (26) }\end{array}$ \\
\hline \multicolumn{3}{|l|}{ AG insaturados trans isômeros } \\
\hline $\begin{array}{l}\text { AG C18:1t } \\
\text { AG C18:2t }\end{array}$ & $\begin{array}{l}\text { Margarinas de consistência firme e gorduras vegetais } \\
\text { parcialmente hidrogenadas } \\
\text { Em pequenas quantidades em carnes, produtos cárneos e lácteos }\end{array}$ & $\begin{array}{l}\uparrow \text { Colesterol total, } \uparrow \text { relação LDL/HDL, } \uparrow \text { triglicerídeos } \\
\uparrow \text { Lipoproteína (a) }\end{array}$ \\
\hline \multicolumn{3}{|l|}{ AG poli-insaturados (AGPIs) } \\
\hline $\begin{array}{l}\text { Família } \omega-3 \\
\text { AG linolênico (C18:3 } \omega 3) \\
\text { - AG essencial }\end{array}$ & $\begin{array}{l}\text { Óleos de soja e canola, linhaça (semente e óleo) e em pequenas } \\
\text { quantidades na carne de frango }\end{array}$ & Precursor dos demais AGPIs da família $\omega 3$ \\
\hline $\begin{array}{l}\text { AG EPA }(\mathrm{C} 20: 5 \omega 3) \\
\text { AG DHA }(C 22: 6 \omega 3)\end{array}$ & $\begin{array}{l}\text { Peixes gordurosos (sardinha), óleos de peixe, em algumas } \\
\text { carnes e ovos (em pequenas quantidades) }\end{array}$ & $\begin{array}{l}\downarrow \text { VLDL colesterol, } \downarrow \text { triglicerídeos } \\
\text { Precursores de componentes anti-inflamatórios (27) } \\
\text { Efeitos benéficos na atividade antitrombótica (28) }\end{array}$ \\
\hline $\begin{array}{l}\text { Família } \omega \text { 6: } \\
\text { AG linoleico (C18:2 } \omega 6 \text { ): } \\
\text { - representante mais abundante na } \\
\text { dieta; AG essencial }\end{array}$ & Óleos vegetais (girassol, milho, arroz, soja), nozes e sementes & $\begin{array}{l}\downarrow \text { Colesterol total, } \downarrow \text { LDL e HDL quando substituem os AGSs } \\
\text { na dieta } \\
\text { Precursores de componentes inflamatórios (27) }\end{array}$ \\
\hline \multicolumn{3}{|l|}{ AG monoinsaturados } \\
\hline $\begin{array}{l}\text { AG oleico (C18:1 } \omega \text { 9): } \\
\text { - representante mais abundante na dieta }\end{array}$ & Óleos de oliva e canola, nozes, amendoim e castanhas & $\begin{array}{l}\downarrow \text { Colesterol total, } \downarrow \text { LDL quando substituem os AGSs na } \\
\text { dieta; não altera HDL }\end{array}$ \\
\hline
\end{tabular}

LDL: Iow-density lipoprotein cholesterol; HDL: high-density lipoprotein cholesterol; AG: ácido graxo; EPA: ácido graxo eicosapentaenoico; DHA: ácido graxo docosa-hexaenoico.

oliva (principal fonte de gordura dessa dieta) e pequena quantidade de lácteos e carne vermelha. Frango e peixes são as fontes proteicas mais consumidas. Ovos estão presentes no máximo quatro unidades por semana, e o vinho tinto é consumido em quantidade moderada (37). O seguimento de dietas do tipo mediterrâneo reduz o colesterol total, LDL e apolipoproteína B e está associado à melhora da função endotelial (38). Este último efeito pode estar relacionado à oferta aumentada de AGPIs $\omega-3$ decorrente do consumo de peixes e óleo de canola (com redução da razão AGPIs $\omega s-6: 3$ e menor atividade inflamatória) (39) e à presença de polifenóis antioxidantes oriundos do vinho e do óleo de oliva (40). A presença do AG oleico na dieta mediterrânea, um AGMI, já foi relacionada à melhora da função endotelial, por promover o deslocamento seletivo de AGSs das membranas celulares - efeito de menor magnitude do que o dos AGPIs (41). Uma ação antitrombogênica da dieta mediterrânea foi demonstrada em um ensaio clínico que durou dois meses e foi realizado em pacientes com DMT2. Quando o AG linoleico ( $\omega-6)$ foi substituído pelo AG oleico (AGMI), ocorreu uma redução de resistência à insulina e restauração da vasodilatação endotélio-dependente (42).

Com base nessas observações, a American Diabetes Association elaborou recomendações em relação à ingestão de gorduras na dieta para pacientes com DM (2). Resumidamente: limitar o consumo de AGSs em 
até 7\% das calorias ingeridas e de colesterol da dieta em até $200 \mathrm{mg}$ ao dia; consumir o mínimo de AGs insaturados trans-isômeros e AGSs e consumir duas ou mais porções de peixe por semana para fornecer AGPIs $\omega$-3. Tais recomendações são semelhantes às preconizadas para tratamento de dislipidemia na população em geral (43).

\section{EFEITOS DOS LIPÍDEOS DA DIETA SOBRE A FUNÇÃO RENAL}

A ingestão de gorduras, induzindo modificações no perfil dos lipídeos séricos e dos possíveis efeitos desses sobre a função renal (ver tópico anterior), pode atuar como um fator associado ao desenvolvimento e progressão da ND. Dietas habituais, com diferentes composições de gorduras, podem representar proteção ou risco para a ND. Modificações do conteúdo de gordura da dieta, por meio de substituição de alimentos ou de suas fontes, de nutrientes específicos ou ainda de suplementos já demonstraram efeitos benéficos sobre a albuminúria em pacientes com DM.

O efeito deletério dos AGSs da dieta na função renal pode ser mediado, em parte, pela consequente elevação do colesterol sérico após o aumento de sua ingestão com concomitante redução da ingestão de AGPIs. A hipercolesterolemia atuaria como fator de risco para o desenvolvimento da ND (20). Além disso, os AGSs podem também induzir diretamente à disfunção endotelial $(15,16)$, que, por sua vez, está associada à microalbuminúria (17). De fato, a função endotelial é modulada diretamente por fatores dietéticos, especialmente pelos AGPIs $\omega$-3 (29) que têm potencial efeito benéfico sobre a doença cardiovascular. Além disso, a gordura da dieta, tanto o tipo quanto a quantidade, podem influenciar processos inflamatórios (27) e estados de doença a eles relacionados (44).

Na revisão sobre os efeitos da gordura da dieta sobre a função renal em pacientes com ND foram encontrados ensaios clínicos e estudos observacionais selecionados no MedLine e Lilacs (língua portuguesa, inglesa e espanhola), além de publicações específicas da área médica e de nutrição até fevereiro de 2008 (descritores utilizados: lipid, fat, fatty acid, diet OR dietary AND diabetic nephropathy, microalbuminuria $O R$ renal disease $A N D$ diabetes). Foram selecionados artigos que avaliaram efeitos sobre a ND de: lipídeos da dieta, suplementação de lipídeos ou dietas com alimentos com composição lipídica diferenciada. Não foram incluídos estudos que avaliaram exclusivamente efeitos de restrição proteica na ND. Entre os 15 estudos selecionados, cinco foram de associação de lipídeos dietéticos com albuminúria, um foi de associação com hiperfiltração glomerular e oito foram ensaios clínicos (Tabelas 2 e 3 ).

\section{Estudos de associação}

Na tabela 2, em ordem cronológica de publicação, estão descritas as principais características dos estudos observacionais nos quais foi demonstrada a associação de lipídeos dietéticos com o desenvolvimento e/ou progressão da ND. A maioria foi realizada em pacientes com DMTl com normo e microalbuminúria $(6-8,45)$, sendo três estudos transversais, um estudo de casos e controles e dois estudos prospectivos. Apenas dois estudos incluíram pacientes com DMT2 $(9,10)$, sendo que o estudo prospectivo incluiu pacientes macroalbuminúricos (9). Os instrumentos de avaliação de consumo alimentar foram: históricos alimentares, recordatórios com ou sem pesagem de alimentos, diários alimentares ou questionários de frequência alimentar. Somente três estudos avaliaram o impacto da dieta no perfil lipídico sérico dos pacientes $(7,9,10)$.

No final da década de 1980, foi descrita uma associação positiva de ingestão de gorduras totais com a microalbuminúria (6). Posteriormente, foi também demonstrada uma associação do maior consumo de AGSs com a presença de microalbuminúria (7), em especial com o ácido mirístico (8). Recentemente, a ingestão diminuída de AGPIs foi associada com microalbuminúria (10). Também a ingestão aumentada de AGPIs e diminuída de AGSs foi associada à regressão para estágios menos avançados da ND em um estudo prospectivo (9). A maior ingestão de gorduras totais e de AGSs foi associada à hiperfiltração glomerular (> $\left.137 \mathrm{~mL} \cdot \mathrm{min}^{-1}\right)$ (45), sendo o aumento da TFG considerado um possível fator de risco para a ND (1).

\section{Ensaios clínicos}

Na tabela 3, em ordem cronológica de publicação, estão resumidos oito ensaios clínicos randomizados que avaliaram os efeitos da modificação da ingestão de lipídeos sobre a ND. Além da modificação do tipo de gordura consumida (tipo de óleo para o preparo dos alimentos e coberturas para passar no pão) (46-48), foram incluídos estudos nos quais a manipulação da gordura ocorreu pela substituição do tipo de carne (carne vermelha por 


\begin{tabular}{|c|c|c|c|c|c|c|c|c|}
\hline \multirow[b]{2}{*}{ Estudo } & \multirow[b]{2}{*}{ Origem } & \multirow[b]{2}{*}{ n } & \multirow[b]{2}{*}{ Pacientes } & \multirow[b]{2}{*}{ Delineamento } & \multirow[b]{2}{*}{$\begin{array}{l}\text { Avaliação } \\
\text { de dieta }\end{array}$} & \multirow[b]{2}{*}{$\begin{array}{l}\text { Tipo de gordura } \\
\text { (expresso como) }\end{array}$} & \multicolumn{2}{|c|}{ Resultados } \\
\hline & & & & & & & Efeito na ND & $\begin{array}{l}\text { Efeito nos lipídeos } \\
\text { séricos }\end{array}$ \\
\hline $\begin{array}{l}\text { Watts e } \\
\text { cols. (6) }\end{array}$ & UK & 30 & $\begin{array}{c}\text { DMT1 } \\
\text { NORMO/MICRO } \\
(1: 1)\end{array}$ & Casos e controles & $\begin{array}{l}\text { Históricos } \\
\text { alimentares } \\
7 \text { dias }\end{array}$ & $\begin{array}{c}\text { Gordura total } \\
\text { (g e \% de energia) }\end{array}$ & $\begin{array}{c}\text { MICRO versus NORMO: } \\
\uparrow \text { Consumo de gorduras } \\
\text { total > nos MICR0: } \\
138,4 \pm 11,5 \text { versus } 94,4 \\
\pm 5,7 \mathrm{~g} / \text { dia } \\
44,4 \pm 1,3 \text { versus } 39,5 \pm \\
1,2 \% \\
\text { de energia }\end{array}$ & Não avaliado \\
\hline $\begin{array}{l}\text { Bouhanick e } \\
\text { cols. (45) }\end{array}$ & França & 110 & $\begin{array}{c}\text { DMT1 } \\
\text { Não proteinúricos }\end{array}$ & Transversal & $\begin{array}{l}\text { Recordatório } \\
\text { alimentar } \\
1 \text { dia }\end{array}$ & $\begin{array}{l}\text { Gordura total } \\
\text { (g/kg de peso } \\
\text { corporal) }\end{array}$ & $\begin{array}{l}\text { Associação com } \\
\text { hiperfiltração glomerular: } \\
\text { Regressão linear } \\
\text { multivariada: } r=0,25 \\
\text { (idade e gordura) }\end{array}$ & Não avaliado \\
\hline $\begin{array}{l}\text { Riley e } \\
\text { Dwyer (7) }\end{array}$ & Austrália & 178 & $\begin{array}{c}\text { DMT1 } \\
\text { NORMO/MICRO }\end{array}$ & $\begin{array}{c}\text { Transversal de } \\
\text { base populacional }\end{array}$ & $\begin{array}{l}\text { Questionário de } \\
\text { frequência } \\
\text { alimentar } \\
152 \text { itens }\end{array}$ & $\begin{array}{c}\text { AGSs } \\
\text { categorizado em } \\
\text { quintis } \\
\text { (\% de energia) }\end{array}$ & $\begin{array}{c}\text { Associação com } \\
\text { microalbuminúria: } \\
\text { Maior quintil }(19,2 \%) \\
\text { versus menor quintil } \\
(13,1 \%) \\
\text { Análise multivariada } \\
\text { (ajustada HDL): } \\
\text { RC = 4,9 (IC } 95 \%=1,2 \\
-20,0)\end{array}$ & $\begin{array}{l}\text { HDL dos MICRO > } \\
\text { NORMO: } \\
61,5 \pm 16,6 \text { versus } \\
53,4 \pm 15,9 \mathrm{mg} / \mathrm{dL}\end{array}$ \\
\hline $\begin{array}{l}\text { Holler e } \\
\text { cols. (8) }\end{array}$ & Alemanha & 37 & $\begin{array}{l}\text { DMT1 } \\
\text { MICR0 }\end{array}$ & $\begin{array}{c}\text { Coorte } \\
\text { prospectivo } \\
\text { Seguimento = } \\
5 \text { anos }\end{array}$ & $\begin{array}{l}\text { Recordatórios } \\
\text { alimentares } \\
4 \text { dias } \\
\text { bimestrais }\end{array}$ & $\begin{array}{l}\text { Gordura total (g) } \\
\text { AGs mirístico, } \\
\text { araquidônico e } \\
\text { linoleico (mg) }\end{array}$ & $\begin{array}{c}\text { Associação com } \\
\text { albuminúria: } \\
\text { Positiva: } A G S, A G \\
\text { mirístico, } A G \\
\text { arquidônico } \\
\text { Negativa: } A G \text { linoleico } \\
\text { Regressão múltipla: } R^{2}= \\
0,589\end{array}$ & Não avaliado \\
\hline $\begin{array}{l}\text { Cárdenas e } \\
\text { cols. } \\
\text { GSEDNu (9) }\end{array}$ & Espanha & 192 & $\begin{array}{c}99 \text { DMT2 } \\
93 \text { DMT2 } \\
\text { NORMO, MICRO, } \\
\text { MACRO }\end{array}$ & $\begin{array}{c}\text { Coorte de base } \\
\text { populacional } \\
\text { Seguimento = } \\
7 \text { anos }\end{array}$ & $\begin{array}{l}\text { Diários alimentares } \\
7 \text { dias } \\
\text { Início e final do } \\
\text { estudo }\end{array}$ & $\begin{array}{l}\text { AGSs, AGPIs e } \\
\text { AGMIs } \\
\text { (\% de energia) }\end{array}$ & $\begin{array}{c}\text { Algum grau de regressão } \\
\text { de estágio de ND } \\
\text { quando: } \\
\text { Ingestão } \uparrow \text { AGPIs e } \downarrow \\
\text { AGSs } \\
\text { Progressão da ND } \\
\text { quando: } \\
\text { Ingestão } \downarrow \text { AGPIs e } \uparrow \\
\text { AGSs }\end{array}$ & $\begin{array}{c}\downarrow \text { colesterol total e } \\
\text { LDL nos pacientes que } \\
\text { regrediram } \\
\downarrow \text { TG séricos mais } \\
\text { acentuada nos } \\
\text { pacientes que } \\
\text { regrediram: } \\
-12,2 \text { versus }-4,6 \%\end{array}$ \\
\hline $\begin{array}{l}\text { Almeida e } \\
\text { cols. (10) }\end{array}$ & Brasil & 181 & $\begin{array}{c}\text { DMT2 } \\
\text { NORMO e MICRO }\end{array}$ & Transversal & $\begin{array}{l}\text { Recordatórios } \\
\text { alimentares com } \\
\text { pesagem de } \\
\text { alimentos } \\
3 \text { dias }\end{array}$ & $\begin{array}{l}\text { AGPIs, AGPIs } \omega-6 \\
\text { Fontes alimentares } \\
\text { de gorduras: óleos } \\
\text { vegetais e manteiga } \\
\text { (nutrientes: \% de } \\
\text { energia) } \\
\text { (alimentos: } \mathrm{mg} / \mathrm{kg} \\
\text { ou g) }\end{array}$ & $\begin{array}{l}\text { Análise multivariada: } \\
\text { Associação positiva com } \\
\text { microalbuminúria: } \\
\text { Manteiga: } R C=1,39 \\
\text { (IC 95\% = 1,03-1,86) } \\
\text { Associação negativa com } \\
\text { microalbuminúria: } \\
\text { AGPIs: RC = 0,86 } \\
\text { (IC 95\% = 0,76-0,96); } \\
\text { AGPIs de origem vegetal: } \\
\text { RC = 0,87 (IC 95\% = } \\
\text { 0,79-0,97); } \\
\text { AGPIs } \omega 6 \text { : } \\
\text { RC = 0,82 (IC 95\% = } \\
0,72-0,93) ; \\
\text { Óleos vegetais: } \\
\text { RC = 0,04 (0,01-0,52) }\end{array}$ & $\begin{array}{c}\text { Sem diferença entre os } \\
\text { pacientes NORMO e } \\
\text { MICRO }\end{array}$ \\
\hline
\end{tabular}

DM: diabetes melito; NORMO: normoalbuminúricos; MICRO: microalbuminúricos; AGSs: ácidos graxos saturados; RC: razão de chances; HDL: high-density lipoprotein cholesterol; AGs: ácidos graxos; MACRO: macroalbuminúricos; AGPIs: ácidos graxos poli-insaturados; AGMIs: ácidos graxos monoinsaturados; ND: nefropatia diabética; TG: triglicerídeos. 
Tabela 3. Ensaios clínicos: manipulação das gorduras na dieta e efeitos na ND

\begin{tabular}{|c|c|c|c|c|c|c|c|}
\hline \multirow[t]{2}{*}{ Estudo } & \multirow[t]{2}{*}{$\mathbf{n}$} & \multirow[t]{2}{*}{ Pacientes } & \multirow{2}{*}{$\begin{array}{l}\text { Delineamento e } \\
\text { tempo de } \\
\text { intervenção }\end{array}$} & \multirow[t]{2}{*}{ Intervenção } & \multirow[t]{2}{*}{ Controle } & \multicolumn{2}{|c|}{ Resultados } \\
\hline & & & & & & Efeito na ND & $\begin{array}{l}\text { Efeito no perfil } \\
\text { lipídico }\end{array}$ \\
\hline $\begin{array}{l}\text { Hamazaki e } \\
\text { cols. (49) }\end{array}$ & 26 & $\begin{array}{l}\text { DMT1 e DMT2: } \\
\text { MICRO }\end{array}$ & $\begin{array}{c}\text { ECR } \\
\text { Tempo = } 6 \text { meses } \\
\text { (cada dieta) }\end{array}$ & $\begin{array}{c}\text { Dieta usual + } \\
\text { suplemento de AGPIs } \\
\text { w3 } 3(1.800 \mathrm{mg} / \mathrm{dia})\end{array}$ & Placebo & $\begin{array}{c}\downarrow 45 \% \text { na excreção } \\
\text { urinária de albumina; } \\
\text { Sem alteração de } \\
\text { glicose, teste A1C e } \\
\text { pressão arterial }\end{array}$ & Ausente \\
\hline
\end{tabular}

\begin{tabular}{|c|c|c|c|c|c|c|c|}
\hline $\begin{array}{l}\text { Dullaart e } \\
\text { cols. (46) }\end{array}$ & 36 & $\begin{array}{c}\text { DMT1: } \\
\text { Excreção urinária de } \\
\text { albumina de } 10 \text { a } 200 \\
\mu \mathrm{g} / \mathrm{min}\end{array}$ & $\begin{array}{c}\text { ECR } \\
\text { Tempo }=2 \text { anos } \\
\text { (cada dieta) }\end{array}$ & $\begin{array}{c}\text { Dieta usual com: } \\
\text { AG linoleico (12\% de } \\
\text { energia) e razão } \\
\text { AGPIs:AGSs = 1,0 }\end{array}$ & $\begin{array}{c}\text { Dieta usual com: } \\
\text { AG linoleico ( } 7 \% \text { de } \\
\text { energia) e } \\
\text { razão AGPIs: AGSs = } \\
0,6\end{array}$ & $\begin{array}{l}\uparrow 58 \% \text { na excreção } \\
\text { urinária de albumina }\end{array}$ & $\begin{array}{c}\downarrow \text { LDL e } \\
\text { apolipoproteína B } \\
\text { após intervenção } \\
\downarrow \text { HDL na intervenção } \\
\text { e controle }\end{array}$ \\
\hline $\begin{array}{l}\text { Pecis e cols. } \\
\text { (13) }\end{array}$ & 15 & $\begin{array}{l}\text { DMT1 NORMO: } \\
9 \text { normofiltrantes } \\
6 \text { hiperfiltrantes }\end{array}$ & $\begin{array}{c}\text { ECR cruzado } \\
\text { Tempo = } 3 \text { semanas } \\
\text { (cada dieta) }\end{array}$ & $\begin{array}{l}\text { 1. Dieta } \\
\text { normoproteica à base } \\
\text { de carne branca } \\
\text { (galinha e peixe) } \\
\text { 2. Dieta hipoproteica }\end{array}$ & $\begin{array}{c}\text { Dieta usual (carne } \\
\text { vermelha) }\end{array}$ & $\begin{array}{c}\downarrow \text { TFG após dieta } \\
\text { normoproteica à base } \\
\text { de carne branca e da } \\
\text { dieta hipoproteica }\end{array}$ & $\begin{array}{c}\text { Benefício somente } \\
\text { nos normofiltrantes: } \\
\downarrow \text { colesterol total e } \\
\text { HDL na dieta } \\
\text { hipoproteica }\end{array}$ \\
\hline $\begin{array}{l}\text { Shimizu e } \\
\text { cols. (50) }\end{array}$ & 45 & $\begin{array}{c}\text { DMT2: } \\
\text { MICRO e MACR0 } \\
\text { (índice albumina/ } \\
\text { creatinina em amostra } \\
\text { urinária) }\end{array}$ & $\begin{array}{c}\text { ECR } \\
\text { Tempo = } 12 \text { meses } \\
\text { (cada dieta) }\end{array}$ & $\begin{array}{l}\text { Suplemento de AGPIs } \\
\qquad \begin{array}{l}\omega 3 \\
(900 \mathrm{mg} / \mathrm{dia} \text { de EPA) }\end{array}\end{array}$ & Placebo & $\begin{array}{c}\text { 凶58\% da albuminúria } \\
\text { aos } 3 \text { meses e } \\
\text { mantém até } 12 \text { meses } \\
\text { Sem alteração de teste } \\
\text { A1C e pressão arterial }\end{array}$ & Ausente \\
\hline $\begin{array}{l}\text { Gross e } \\
\text { cols. (11) }\end{array}$ & 28 & $\begin{array}{c}\text { DMT2: } \\
15 \text { NORMO } \\
13 \text { MICR0 }\end{array}$ & $\begin{array}{c}\text { ECR cruzado } \\
\text { Tempo = } 4 \text { semanas } \\
\text { (cada dieta) }\end{array}$ & $\begin{array}{l}\text { 1. Dieta } \\
\text { normoproteica à base } \\
\text { de carne de galinha } \\
\text { 2. Dieta hipoproteica }\end{array}$ & $\begin{array}{l}\text { Dieta usual (carne } \\
\text { vermelha) }\end{array}$ & $\begin{array}{c}\text { NORMO: } \\
\downarrow \text { TFG após a dieta à } \\
\text { base de galinha e } \\
\text { dieta hipoproteica } \\
\text { MICR0: } \\
\text { Maior } \downarrow \text { excreção } \\
\text { urinária de albumina } \\
\text { após dieta à base de } \\
\text { galinha (46\%) } \\
\text { quando comparada } \\
\text { com dieta } \\
\text { hipoproteica (18\%) e } \\
\text { dieta usual }\end{array}$ & $\begin{array}{c}\text { Benefício somente } \\
\text { nos MICRO: } \\
\downarrow \text { apolipoproteína B e } \\
\text { colesterol total após } \\
\text { dieta à base de } \\
\text { galinha e da } \\
\text { hipoproteica }\end{array}$ \\
\hline $\begin{array}{l}\text { Barnard e } \\
\text { cols. (47) }\end{array}$ & 99 & $\begin{array}{c}\text { DMT2: } \\
79 \text { NORMO } \\
20 \text { MICRO }\end{array}$ & $\begin{array}{c}\text { ECR } \\
\text { Tempo }=22 \text { semanas } \\
\text { (cada dieta) }\end{array}$ & $\begin{array}{c}\text { Dieta vegetariana } \\
\text { restrita: } \\
15 \% \text { de proteínas } \\
10 \% \text { de gordura total: } \\
<5 \% \text { de AGSs e } \\
<50 \text { mg de colesterol }\end{array}$ & $\begin{array}{c}\text { Dieta preconizada } \\
\text { pela ADA: } \\
\text { 15\%-20\% proteínas } \\
7 \% \text { de AGSs } \\
60 \%-70 \% \text { de } \\
\text { carboidratos e AGMls } \\
<200 \text { mg de } \\
\text { colesterol }\end{array}$ & $\begin{array}{c}\text { Maior } \downarrow \text { excreção } \\
\text { urinária de albumina } \\
\text { após dieta vegetariana } \\
\text { restrita }(56 \%) \text { quando } \\
\text { comparada com dieta } \\
\text { ADA }(21 \%)\end{array}$ & $\begin{array}{c}\downarrow \text { LDL após dieta } \\
\text { vegetariana restrita e } \\
\text { dieta preconizada pela } \\
\text { ADA }\end{array}$ \\
\hline $\begin{array}{l}\text { De Mello e } \\
\text { cols. (12) }\end{array}$ & 17 & $\begin{array}{l}\text { DMT2: } \\
\text { MACRO }\end{array}$ & $\begin{array}{c}\text { ECR cruzado } \\
\text { Tempo = } 4 \text { semanas } \\
\text { (cada dieta) }\end{array}$ & $\begin{array}{l}\text { 1. Dieta hipoproteica } \\
\text { 2. Dieta } \\
\text { normoproteica à base } \\
\text { de carne de galinha }\end{array}$ & $\begin{array}{c}\text { Dieta usual (carne } \\
\text { vermelha) }\end{array}$ & $\begin{array}{c}\downarrow 20,6 \% \text { da excreção } \\
\text { urinária de albumina } \\
\text { após dieta à base de } \\
\text { galinha e } \\
\downarrow 31,4 \% \text { da excreção } \\
\text { urinária de albumina } \\
\text { após dieta } \\
\text { hipoproteica }\end{array}$ & $\begin{array}{c}\downarrow \text { Colesterol não-HDL } \\
\text { e } \uparrow \text { AGPIs séricos } \\
\text { após dietas à base de } \\
\text { galinha e hipoproteica } \\
\downarrow \text { TG e AG palmítico } \\
\text { sérico após dieta à } \\
\text { base de galinha }\end{array}$ \\
\hline $\begin{array}{l}\text { Yamamoto e } \\
\text { cols. (48) }\end{array}$ & 15 & $\begin{array}{c}\text { DMT2: } \\
\text { MACR0 } \\
\text { (creatinina sérica } \\
0,48-4,84 \mathrm{mg} / \mathrm{dl} \text { ) }\end{array}$ & $\begin{array}{c}\text { ECR } \\
\text { Tempo = } 6 \text { meses } \\
\text { (cada dieta) }\end{array}$ & $\begin{array}{l}\text { Dieta usual com } 10 \mathrm{~g} \\
\text { de óleo rico em DAGs }\end{array}$ & $\begin{array}{c}\text { Dieta usual com } 10 \mathrm{~g} \\
\text { de óleo habitual (rico } \\
\text { em TAGs) }\end{array}$ & $\begin{array}{l}\text { Menor perda de } \\
\text { função renal } \\
\text { (manutenção da } \\
\text { creatinina sérica) na } \\
\text { dieta com DAGs }\end{array}$ & $\begin{array}{c}\downarrow \text { TG na dieta com } \\
\text { DAGs }\end{array}$ \\
\hline
\end{tabular}

DM: diabetes melito; MICRO: microalbuminúricos; ECR: ensaio clínico randomizado; AGPIs: ácidos graxos poli-insaturados; excreção urinária de albumina: excreção urinária de albumina; AG: ácido graxo; AGSs: ácidos graxos saturados; LDL: Iow-density lipoprotein cholesterol; HDL: high-density lipoprotein cholesterol; NORMO: normoalbuminúricos; TFG: taxa de filtração glomerular; MACRO: macroalbuminúricos; EPA: ácido graxo eicosapentaenoico; ADA: American Diabetes Association; TG: triglicerídeos; DAGs: diacilgliceróis; TAGs: triacilgliceróis. 
carne de galinha) (12-14) ou ainda pela suplementação na dieta usual com AGPIs $\omega-3$ (49,50). O tempo médio de intervenção dietética dos estudos variou de três semanas a 24 meses, e, quando o delineamento foi um ensaio clínico randomizado cruzado (11-13), o período de washout entre as dietas foi de três a quatro semanas.

Efeitos benéficos sobre a ND com redução da excreção urinária de albumina foram demonstrados com a adoção de dieta vegetariana pobre em gordura (47), com substituição de óleos ricos em triacilgliceróis por diacilgliceróis (48) e com substituição da carne vermelha da dieta por carne de galinha $(11,12)$ e suplementação com AGPIs $\omega$-3 $(49,50)$. Com a dieta baseada em carne de galinha foi também observado um efeito benéfico sobre a hiperfiltração glomerular (13). Alguns autores demonstraram também uma melhora concomitante no perfil lipídico, tanto em pacientes com microalbuminúria $(11,47)$ quanto naqueles com macroalbuminúria $(12,48)$. Em um único estudo, após o acréscimo da ingestão de ácido linoleico ( $\omega$-6), a excreção urinária de albumina aumentou em $58 \%$, embora tenha ocorrido uma melhora do perfil lipídio sérico (46). Uma explicação para esse resultado não esperado sobre a excreção urinária de albumina seriam os efeitos distintos dos AGPIs $\omega$-6 e $\omega$-3 no organismo, já que os AGs $\omega$-3 reduzem a excreção urinária de albumina $(49,50)$.

Em relação aos efeitos benéficos da redução de gorduras da dieta sobre a excreção urinária de albumina, a influência da diminuição concomitante de proteínas não pode ser desconsiderada. Como exemplo há o estudo sobre a adoção de dieta vegetariana pobre em gordura (47). Entretanto, é provável que a gordura da dieta, em especial os AGs, tenha um papel independente sobre a função renal. Reforça essa afirmação a demonstração de que, em pacientes com DMT2, uma dieta à base de carne de galinha, sem restrição de proteínas, reduz a albuminúria mais do que a dieta com restrição proteica (12).

Conclui-se que, de maneira geral, a manipulação de gorduras específicas da dieta é capaz de trazer benefícios sobre a excreção urinária de albumina, idealmente em associação com uma melhora do perfil lipídico. Entretanto, deve ser lembrado que os estudos que mostram esses efeitos são de curta duração, avaliam um número de paciente relativamente limitado e que não há evidências contundentes de benefícios sobre desfechos clínicos definitivos como a falência renal e/ou morte. Não existem ainda recomendações definitivas para manipulação das gorduras da dieta com vistas à proteção da função renal na ND.

\section{INTERAÇÃO GENÉTICA: GORDURAS DA DIETA E PERFIL LIPÍDICO}

A influência de fatores genéticos na presença da ND tem sido demonstrada (5l). É provável que a suscetibilidade genética para a presença de valores anormais de albuminúria e de TFG (doença renal terminal) seja distinta (52). Aspectos genéticos relacionados à patogênese da ND podem também estar associados a fatores dietéticos que levem às alterações nos aos lipídeos séricos. Polimorfismos genéticos explicariam a variabilidade interindividual da resposta de lipoproteínas aos componentes dietéticos (53).

Para a revisão de artigos relacionados a polimorfismos possivelmente associados à dislipidemia ou aos lipídeos da dieta na ND, foram utilizados o MedLine e o Lilacs (língua portuguesa, inglesa e espanhola), além de publicações específicas da área até fevereiro de 2008. Os descritores utilizados foram: "polymorphism" AND "diabetic nephropathy", "microalbuminuria" OR "renal disease” AND “diabetes”. Quinze estudos foram selecionados: um sobre o gene que modula a proteína intestinal carreadora de AGs (I-FABP, intestinal fatty acid binding protein), nove estudos sobre as variações do gene da apolipoproteína $\mathrm{E}$ (apoE) e quatro sobre o gene que modula o receptor ativado por proliferadores do peroxissoma (PPAR $\gamma 2$, peroxisome proliferatoractivated receptor- $\gamma 2$ ). Dois estudos foram transversais, 11 de casos e controles e um prospectivo (4,5 anos de acompanhamento). Os estudos foram realizados em diferentes populações, com pacientes com DM Tl $(54,55)$ e com DMT2 (56-67). A maioria deles observou uma associação positiva com a ND $(54,56,57,59,60,62,63)$. As descrições das características gerais dos polimorfismos genéticos associados aos lipídeos em estudos com pacientes com ND estão resumidas na tabela 4 .

Os seguintes polimorfismos foram associados com a presença e/ou progressão da ND: o Ala54Thr do gene do FABP2 (62) e o polimorfismo da apoE (alelo $\in 2$ ) $(54,56,57)$. Já uma associação negativa, portanto representando uma provável proteção, foi demonstrada para o polimorfismo Ala12Pro do gene do PPAR $\gamma 2$, tanto para microalbuminúria $(58)$ quanto para estágios mais avançados de ND $(61,67)$. No caso do polimorfismo da $a p o E$, a associação com a ND ainda é controversa quando o alelo presente for $o \in 4$ (55,63-65).

Em conclusão, as alterações genéticas parecem modificar os efeitos das gorduras da alimentação sobre o perfil lipídico e, assim, influenciar o desenvolvimento e 


\begin{tabular}{|c|c|c|c|c|c|c|c|}
\hline \multicolumn{2}{|c|}{ Polimorfismo } & \multicolumn{2}{|c|}{ Lipoproteína ou receptor relacionado } & \multirow{2}{*}{$\begin{array}{c}\text { Efeito do } \\
\text { polimorfismo no } \\
\text { metabolismo lipídico }\end{array}$} & \multirow{2}{*}{$\begin{array}{l}\text { Associação } \\
\text { na ND }\end{array}$} & \multicolumn{2}{|r|}{ Estudo } \\
\hline Gene & Descrição & $\begin{array}{l}\text { Lipoproteína ou } \\
\text { receptor }\end{array}$ & Ação & & & $\mathbf{n}$ & Pacientes \\
\hline$F A B P 2$ & $\begin{array}{l}\text { Substituição de uma } \\
\text { alanina por uma } \\
\text { treonina (Ala54Thr) } \\
\text { no códon } 54 \\
\text { (cromossomo 4): } \\
\text { Genótipos: AlaAla, } \\
\text { ThrThr, AlaThr } \\
\text { Alelo de risco = Thr }\end{array}$ & $\begin{array}{l}\text { Proteína intracelular } \\
\text { expressa no intestino } \\
\text { (I-FABP) }\end{array}$ & $\begin{array}{l}\text { Carreadora de AGs } \\
\text { longos da dieta }\end{array}$ & $\begin{array}{c}\text { Pacientes com alelo Thr: } \\
\uparrow \text { TG séricos em } \\
\text { pacientes DMT2 (68) }\end{array}$ & Positiva & 1.042 & $\begin{array}{l}\text { DMT2, brasileiros } \\
\text { Transversal, } \\
\text { multicêntrico (62) }\end{array}$ \\
\hline \multirow[t]{9}{*}{ ApoE } & \multirow{9}{*}{$\begin{array}{c}\text { Variações do gene } \\
\text { produzem três alelos: } \\
\in 2, \in 3 \text { e } \in 4 \\
\text { (cromossomo 19): } \\
\text { Genótipos } \\
\text { homozigotos: E2/2, } \\
\text { E3/3 e E4/4 } \\
\text { Genótipos } \\
\text { heterozigotos: E2/3, } \\
\text { E2/4 e E3/4 }\end{array}$} & \multirow[t]{9}{*}{$\begin{array}{c}\text { Glicoproteína } \\
\text { polimórfica, presente } \\
\text { nas lipoproteínas }\end{array}$} & \multirow{9}{*}{$\begin{array}{l}\text { Mediadora do } \\
\text { receptor de LDL, } \\
\text { atuando no } \\
\text { catabolismo das } \\
\text { lipoproteínas ricas } \\
\text { em TG e no } \\
\text { transporte reverso do } \\
\text { colesterol }\end{array}$} & \multirow{5}{*}{$\begin{array}{c}\text { Pacientes com alelo } \in 2 \text { : } \\
\downarrow \text { resposta do colesterol } \\
\text { total, LDL e HDL após } \\
\text { manipulação das } \\
\text { gorduras da dieta (53) }\end{array}$} & $\begin{array}{l}\text { Possível associação } \\
\text { positiva com } \\
\text { macroalbuminúria }\end{array}$ & 167 & $\begin{array}{c}\text { DMT2 coreanos } \\
\text { Casos e controles (56) }\end{array}$ \\
\hline & & & & & $\begin{array}{c}\text { Positiva para } \\
\text { macroalbuminúria e } \\
\text { diálise }\end{array}$ & 464 & $\begin{array}{l}\text { DMT2, tailandeses } \\
\text { Casos e controles (57) }\end{array}$ \\
\hline & & & & & $\begin{array}{c}\text { Positiva para } \\
\text { progressão dos } \\
\text { estágios de ND }\end{array}$ & 429 & $\begin{array}{c}\text { DMT2, japoneses } \\
\text { Prospectivo de } 4,5 \text { anos } \\
\text { de seguimento (59) }\end{array}$ \\
\hline & & & & & $\begin{array}{l}\text { Positiva para falência } \\
\text { renal (hemodiálise) }\end{array}$ & 419 & $\begin{array}{l}\text { DMT1, americanos } \\
\text { Casos e controles (54) }\end{array}$ \\
\hline & & & & & $\begin{array}{l}\text { Risco aumentado de } \\
\text { albuminúria: } \\
\text { co-herança com } \\
\text { polimorfismo do } \\
\text { gene HSPG }\end{array}$ & 298 & $\begin{array}{c}\text { DMT2, chineses } \\
\text { Casos e controles (60) }\end{array}$ \\
\hline & & & & Todos os alelos & Sem associação & 166 & $\begin{array}{c}\text { DMT1, russos } \\
\text { Casos e controles (55) }\end{array}$ \\
\hline & & & & \multirow{3}{*}{$\begin{array}{l}\text { Pacientes com alelo } \in 4: \\
\uparrow \text { resposta do colesterol } \\
\text { total, HDL e LDL após } \\
\text { manipulação das } \\
\text { gorduras da dieta (53) }\end{array}$} & Positiva para ND & 108 & $\begin{array}{c}\text { DMT2, turcos } \\
\text { Casos e controles (63) }\end{array}$ \\
\hline & & & & & $\begin{array}{c}\text { Negativa para } \\
\text { microalbuminúria }\end{array}$ & 94 & $\begin{array}{c}\text { DMT2, coreanos } \\
\text { Casos e controles (64) }\end{array}$ \\
\hline & & & & & $\begin{array}{l}\text { Negativa para } \\
\text { falência renal } \\
\text { (hemodiálise) }\end{array}$ & 85 & $\begin{array}{c}\text { DMT2, chilenos } \\
\text { Casos e controles (65) }\end{array}$ \\
\hline \multirow[t]{4}{*}{ PPAR $\gamma 2$} & \multirow{4}{*}{$\begin{array}{c}\text { Substituição de uma } \\
\text { alanina por prolina } \\
\text { na posiçãa } 12 \\
\text { (Ala12Pro) no ponto } \\
\text { de mutação no éxon } \\
\text { B da parte NH2 } \\
\text { terminal do PPAR } \gamma 2 \\
\text { Genótipo: AlaAla; } \\
\text { AlaPro; ProPro } \\
\text { Alelo de proteção = } \\
\text { Ala }\end{array}$} & \multirow[t]{4}{*}{$\begin{array}{l}\text { Receptor ativado por } \\
\text { proliferadores do } \\
\text { peroxissoma (PPAR) } \gamma 2\end{array}$} & \multirow{4}{*}{$\begin{array}{c}\text { Atua na diferenciação } \\
\text { de adipócitos; } \\
\downarrow \text { Desensibilização } \\
\text { da ação à insulina } \\
\text { pelos AGs séricos } \\
\text { livres, fator- } \alpha \text { de } \\
\text { necrose tumoral e } \\
\text { resistina e } \uparrow \text { ação da } \\
\text { adiponectina }\end{array}$} & \multirow[t]{4}{*}{$\begin{array}{l}\text { Pacientes com alelo Ala: } \\
\uparrow \text { sensibilidade à ação da } \\
\text { insulina (69) }\end{array}$} & $\begin{array}{l}\text { Negativa para } \\
\text { macroalbuminúria e } \\
\text { falência renal } \\
\text { (hemodiálise) }\end{array}$ & 445 & $\begin{array}{c}\text { DMT2, alemães } \\
\text { Casos e controles (58) }\end{array}$ \\
\hline & & & & & $\begin{array}{l}\text { Sem associação } \\
\text { (éxon } 2 \text { e éxon 6) }\end{array}$ & 141 & $\begin{array}{c}\text { DMT2, turcos } \\
\text { Casos e controles (66) }\end{array}$ \\
\hline & & & & & $\begin{array}{c}\text { Negativa para } \\
\text { proteinúria e falência } \\
\text { renal }\end{array}$ & 316 & $\begin{array}{c}\text { DMT2, brasileiros } \\
\text { Casos e controles (61) }\end{array}$ \\
\hline & & & & & $\begin{array}{l}\text { Negativa para } \\
\text { microalbuminúria }\end{array}$ & 159 & $\begin{array}{l}\text { DMT2, aborígenes } \\
\text { canadenses } \\
\text { Transversal (67) }\end{array}$ \\
\hline
\end{tabular}

ND: nefropatia diabética; I-FABP: intestinal fatty acid binding protein; AG: ácidos graxos; TG: triglicerídeos; DM: diabetes melito; ApoE: apolipoproteína E; LDL: Iow-density lipoprotein cholesterol; HDL: high-density lipoprotein cholesterol; HSPG: heparan sulfate proteoglycane; PPAR: peroxisome proliferator-activated receptor.

a progressão da ND. Entretanto, quase todos os estudos de associação são transversais. São necessários mais estudos de coorte para analisar a evolução da doença re- nal e dieta. Idealmente, deveriam ser realizados ensaios clínicos para avaliar a resposta à ingestão de gordura em pacientes com diferentes genótipos de risco para ND. 


\section{COMENTÁRIOS FINAIS}

As evidências disponíveis sugerem que os lipídeos dietéticos e séricos podem ter papel importante no desenvolvimento e na progressão da ND. Um perfil lipídico sérico desfavorável (valores aumentados de colesterol total, de triglicerídeos, de apolipoproteína B e de colesterol LDL - além de partículas mais densas - com valores diminuídos de colesterol HDL), incluindo alterações de AGs, provavelmente precede à instalação da ND. Essas alterações lipídicas estão relacionadas à ingestão de gorduras. De fato, a composição dos AGs séricos associase à microalbuminúria, à disfunção endotelial e a um padrão alimentar rico em AGSs e pobre em AGPIs. Tal tipo de dieta provavelmente representa um fator de risco para a ND, além do já estabelecido aumento de risco cardiovascular. Embora as evidências atuais sugiram fortemente que a manipulação de gorduras da dieta seja capaz de reduzir a excreção urinária de albumina com concomitante melhora do perfil lipídico, não existe, até o presente momento, uma recomendação específica nesse sentido para prevenção ou tratamento de ND.

Finalmente, cabe salientar a existência da interação entre fatores alimentares e genéticos na resposta intraindividual do perfil lipídico à ingestão de gorduras. A identificação de pacientes com risco aumentado para desenvolvimento ou progressão da ND pela presença de polimorfismos genéticos específicos e suas interrelações permitirá a adoção de estratégias nutricionais individualizadas.

Agradecimentos: este trabalho foi apoiado parcialmente pelo Ministério de Ciência e Tecnologia, Conselho Nacional de Desenvolvimento Científico e Tecnológico (MCT/CNPq \# 502050/2005-5).

Declaração: os autores declaram não haver conflito de interesse científico neste artigo.

\section{REFERÊNCIAS}

1. Gross JL, Azevedo MJ, Silveiro SP, Canani LH, Caramori ML, Zelmanovitz T. Diabetic nephropathy: diagnosis, prevention, and treatment. Diabetes Care. 2005;28(1):164-76.

2. American Diabetes Association. Standard of Medical Care in Diabetes-2008. Diabetes Care. 2008;31:S12-S54.

3. Kramer CK, Leitão CB, Pinto LC, Silveiro SP, Gross JL, Canani LH. Clinical and laboratory profile of patients with type 2 diabetes with low glomerular filtration rate and normoalbuminuria. Diabetes Care. 2007;30(8):1998-2000.

4. Toeller M, Buyken A, Heitkamp G, Brämswig S, Mann J, Milne $R$, et al. Protein intake and urinary albumin excretion rates in the EURODIAB IDDM complications study. Diabetologia. 1997;40(10):1219-26.
5. Möllsten A, Dahlquist GG, Stattin E, Rudberg S. Higher intakes of fish protein are related to a lower risk of microalbuminuria in young Swedish type 1 diabetic patients. Diabetes Care. 2001;24(5):805-10.

6. Watts GF, Gregory L, Naoumova R, Kubal C, Shaw KM. Nutrient intake in insulin-dependent diabetic patients with incipient nephropathy. Eur J Clin Nutr. 1988;42(8):697-702.

7. Riley MD, DwyerT. Microalbuminuria is positively associated with usual dietary saturated fat acid intake and negatively associated with usual dietary protein intake in people with insulin-dependent diabetes mellitus. Am J Clin Nutr. 1998;67(1):50-7.

8. Holler $\mathrm{C}$, Abrahamian $\mathrm{H}$, Auinger $\mathrm{M}$. Effect of nutrition on microalbuminuria in patients with type 1 diabetes: prospective data evaluation over 5 years. Acta Med Austriaca. 1999;26(5):168-72.

9. Cárdenas C, Bordiu E, Bagazgoitia J, Calle-Pascual AL; Diabetes and Nutrition Study Group, Spanish Diabetes Association Polyunsaturated fatty acid consumption may play a role in the onset and regression of microalbuminuria in well-controlled type 1 and type 2 diabetic people: a 7-year, prospective, population-based, observational multicenter study. Diabetes Care. 2004;27(6):1454-7.

10. Almeida JC, Zelmanovitz T, Vaz JS, Steemburgo T, Perassolo MS, Gross JL, et al. Sources of protein and polyunsaturated fatty acids of the diet and microalbuminuria in type 2 diabetes mellitus. $J$ Am Coll Nutr. 2008;27(5):528-37.

11. Gross JL, Zelmanovitz T, Moulin CC, Mello VD, Perassolo M, Leitao $C$, et al. Effect of a chicken-based diet on renal function and lipid profile in patients with type 2 diabetes. Diabetes Care. 2002;25(4):645-51.

12. De Mello VD, ZelmanovitzT, Perassolo MS, Azevedo MJ, Gross JL. Withdrawal of red meat from the usual diet reduces albuminuria and improves serum fatty acid profile in type 2 diabetic patients with macroalbuminuria. Am J Clin Nutr. 2006;83(5):1032-8.

13. Pecis M, de Azevedo MJ, Gross JL. Chicken and fish diet reduces glomerular hyperfiltration in IDDM patients. Diabetes Care. 1994;17(7):665-72.

14. Perassolo MS, Almeida JC, Prá RL, Mello VD, Maia AL, Moulin $\mathrm{CC}$, et al. Fatty acid composition of serum lipid fractions in type 2 diabetic patients with microalbuminuria. Diabetes Care. 2003;26(3):613-8.

15. Fernández-Real JM, Broch M, Vendrell J, Ricart W. Insulin resistance, inflammation, and serum fatty acid composition. Diabetes Care. 2003;26(5):1362-8.

16. Perassolo MS, Almeida JC, Steemburgo T, Dall'Alba V, Mello VDF, Zelmanovitz T, et al. Serum levels of endothelin-1 are related to the serum fatty acid composition in patients with type 2 diabetes. Metabolism. 2008;57(9):1167-72.

17. Zanatta CM, Gerchman F, Burttet L, Nabinger G, Jacques-Silva MC, Canani $\mathrm{LH}$, et al. Endothelin-1 levels and albuminuria in patients with type 2 diabetes mellitus. Diab Res Clin Pract. 80(2):299-304.

18. Thomas $M C$, Rosengård-Bärlund $M$, Mills V, Rönnback $M$, Thomas $\mathrm{S}$, Forsblom C, et al. Serum lipids and the progression of nephropathy in type 1 diabetes. Diabetes Care. 2006;29(2):317-22.

19. Niskanen L, Uusitupa M, Sarlund H, Siitonen O, Voutilainen E, Penttilä I, et al. Microalbuminuria predicts the development of serum lipoprotein abnormalities favoring atherogenesis in newly diagnosed type 2 (non-insulin-dependent) diabetic patients. Diabetologia. 1990;33(4):237-43.

20. Appel GB, Radhakrishnan J, Avram MM, DeFronzo RA, EscobarJimenez F, et al; RENAAL Study. Analysis of metabolic parameters as predictors of risk in the RENAAL Study. Diabetes Care. 2003;26(5):1402-7.

21. Diamond JR. Analogous pathobiologic mechanisms in glomeruIosclerosis and atherosclerosis. Kidney Int Suppl. 1991;39:S29-34. 
22. Goldberg IJ. Clinical review 124: Diabetic dyslipidemia: causes and consequences. J Clin Endocrinol Metab. 2001;86(3):965-71.

23. Panarotto $D$, Rémillard $P$, Bouffard $L$, Maheux $P$. Insulin resistance affects the regulation of lipoprotein lipase in the postprandial period and in an adipose tissue-specific manner. Eur J Clin Invest. 2002;32(2):84-92.

24. Lima DM. Tabela de composição dos alimentos - TACO. 2. Ed. Campinas: Nepa-Unicamp, 2006. Versão II.

25. Schaefer EJ. Lipoproteins, nutrition, and heart disease. Am J Clin Nutr. 2002;75(2):191-212.

26. Baba S, Natsume M, Yasuda A, Nakamura Y, Tamura T, Osakabe $\mathrm{N}$, et al. Plasma LDL and HDL cholesterol and oxidized LDL concentrations are altered in normo- and hypercholesterolemic humans after intake of different levels of cocoa powder. J Nutr. 2007;137(6):1436-41.

27. Simopoulos AP. Essential fatty acids in health and chronic disease. Am J Clin Nutr. 1999;70(3 Suppl):560S-569S.

28. Mutanen M, Freese R. Fats, lipids and blood coagulation. Curr Opin Lipidol. 2001;12(1):25-9.

29. Brown AA, Hu FB. Dietary modulation of endothelial function: implications for cardiovascular disease. Am J Clin Nutr. 2001;73(4):673-86

30. Meydani SN, Dinarello CA. Influence of dietary fatty acids on cytokine production and its clinical implications. Nutr Clin Pract. 1993;8(2):65-72.

31. Hu FB, van Dam RM, Liu S. Diet and risk of type II diabetes: the role of types of fat and carbohydrate. Diabetologia. 2001;44(7):805-17.

32. Wang $C$, Harris WS, Chung M, Lichtenstein AH, Balk EM, Kupelnick B, et al. n-3 Fatty acids from fish or fish-oil supplements, but not alpha-linolenic acid, benefit cardiovascular disease outcomes in primary- and secondary-prevention studies: a systematic review. Am J Clin Nutr. 2006;84(1):5-17.

33. Hartweg J, Farmer AJ, Holman RR, Neil HA. Meta-analysis of the effects of $n-3$ polyunsaturated fatty acids on haematological and thrombogenic factors in type 2 diabetes. Diabetologia. 2007;50(2):250-8.

34. Hartweg J, Farmer AJ, Perera R, Holman RR, Neil HA. Meta-analysis of the effects of $n-3$ polyunsaturated fatty acids on lipoproteins and other emerging lipid cardiovascular risk markers in patients with type 2 diabetes. Diabetologia. 2007;50(8):1593-602.

35. Trichopoulou A, Costacou T, Bamia C, Trichopoulos D. Adherence to a Mediterranean diet and survival in a Greek population. $\mathrm{N}$ Engl J Med. 2003;348(26):2599-608.

36. de Lorgeril M, Salen P, Martin JL, Moujaud I, Delaye J, Mamelle N. Mediterranean diet, traditional risk factors, and the rate of cardiovascular complications after myocardial infarction final report of the Lyon Diet Heart Study. Circulation. 1999;99(6):779-85.

37. Willett WC, Sacks F, Trichopoulou A, Drescher G, Ferro-Luzzi A, Helsing $E$, et al. Mediterranean diet pyramid: a cultural model for healthy eating. Am J Clin Nutr. 1995;61(6 Suppl):S1402-6.

38. Serra-Majem L, Roman B, Estruch R. Scientific Evidence of Interventions using the Mediterranean diet: a systematic review. Nutr Rev. 2006;64(2 Pt 2):S27-47.

39. Ambring A, Johansson M, Axelsen M, Gan L, Strandvik B, Friberg P. Mediterranean-inspired diet lowers the ratio of serum phospholipid n-6 to n-3 fatty acids, the number of leukocytes and platelets, and vascular endothelial growth factor in healthy subjects. Am J Clin Nutr. 2006;83(3):575-81.

40. Carluccio MA, Siculella L, Ancora MA, Massaro M, Scoditti E, Storelli $C$, et al. Olive oil and red wine antioxidant polyphenols inhibit endothelial activation: antiatherogenic properties of Mediterranean diet phytochemicals. Arterioscler Thromb Vasc Biol. 2003;23(4):622-9.

41. Carluccio MA, Massaro $M$, Bonfrate $C$, Siculella $L$, Maffia M, Nicolardi G, et al. Oleic acid inhibits endothelial activation: A di- rect vascular antiatherogenic mechanism of a nutritional component in the Mediterranean diet. Arterioscler Thromb Vasc Biol. 1999;19(2):220-8.

42. Ryan M, Mclnerney D, Owens D, Collins P, Johnson A, Tomkin GH. Diabetes and the Mediterranean diet: a beneficial effect of oleic acid on insulin sensitivity, adipocyte glucose transport and endothelium-dependent vasoreactivity. QJM. 2000;93(2):85-91.

43. Departamento de Aterosclerose da Sociedade Brasileira de Cardiologia. IV Diretriz Brasileira sobre Dislipidemias e Prevenção da Aterosclerose. Arq Bras Cardiol. 2007;88(S1):S2-19.

44. Burr ML, Fehily AM, Gilbert JF, Rogers S, Holliday RM, Sweetnam $P M$, et al. Effects of changes in fat, fish, and fibre intakes on death and myocardial reinfarction: diet and reinfarction trial (DART). Lancet. 1989;2(8666):757-61.

45. Bouhanick B, Suraniti S, Berrut G, Bled F, Simard G, Lejeune JJ et al. Relationship between fat intake and glomerular filtration rate in normotensive insulin-dependent diabetic patients. Diabete Metab. 1995;21(3):168-72.

46. Dullaart RP, Beusekamp BJ, Meijer S, Hoogenberg K, van Doormaal JJ, Sluiter WJ. Long-term effects of linoleic-acid-enriched diet on albuminuria and lipid levels in type 1 (insulin-dependent) diabetic patients with elevated urinary albumin excretion. Diabetologia. 1992;35(2):165-72.

47. Barnard ND, Cohen J, Jenkins DJA, Turner-McGrievy G, Gloede L, Jaster $B$, et al. A low-fat vegan diet improves glycemic control and cardiovascular risk factors in a randomized clinical trial in individuals with type 2 diabetes. Diabetes Care. 2006;29(8):1777-83.

48. Yamamoto K, Tomonobu K, Asakawa H, Tokunaga K, Hase T, Tokimitsu I, et al. Diet therapy with diacylglycerol oil delays the progression of renal failure in type 2 diabetic patients with nephropathy. Diabetes Care. 2006;29(2):417-9.

49. Hamazaki T, Takazakura E, Osawa K, Urakaze M, Yano S. Reduction in microalbuminuria in diabetics by eicosapentaenoic acid ethyl ester. Lipids. 1990;25(9):541-5.

50. Shimizu H, Ohtani K, Tanaka $Y$, Sato N, Mori M, Shimomura $Y$. Long-term effect of eicosapentaenoic acid ethyl (EPA-E) on albuminuria of non-insulin dependent diabetic patients. Diabetes Res Clin Pract. 1995;28(1):35-40.

51. Canani LH, Gershman F, Gross JL. Familial clustering of diabetic nephropathy in Brazilian type 2 diabetic patients. Diabetes. 1999;48(4):909-13.

52. Placha G, Canani LH, Warram JH, Krolewski AS. Evidence for different susceptibility genes for proteinuria and ESRD in type 2 diabetes. Adv Chronic Kidney Dis. 2005;12(2):155-69.

53. Masson LF, McNeill G. The effect of genetic variation on the lipid response to dietary change: recent findings. Curr Opin Lipidol. 2005;16(1):61-7.

54. Araki S, Moczulski DK, Hanna L, Scott LJ, Warram JH, Krolewski AS. APOE polymorphisms and the development of diabetic nephropathy in type 1 diabetes: results of case-control and familybased studies. Diabetes. 2000;49(12):2190-5.

55. Shcherbak NS. Apolipoprotein E gene polymorphism is not a strong risk factor for diabetic nephropathy and retinopathy in Type I diabetes: case-control study. BMC Med Genet. 2001;2:8.

56. Ha SK, Park HS, Kim KW, Kim SJ, Kim DH, Kim JH, et al. Association between apolipoprotein $\mathrm{E}$ polymorphism and macroalbuminuria in patients with non-insulin dependent diabetes mellitus. Nephrol DialTransplant. 1999;14(9):2144-9.

57. Hsieh MC, Lin SR, Yang YC, Chen HC, Lin JN, Shin SJ. Higher frequency of apolipoprotein E2 allele in type 2 diabetic patients with nephropathy in Taiwan. J Nephrol. 2002;15(4):368-73.

58. Herrmann SM, Ringel J, Wang JG, Staessen JA, Brand E; Berlin Diabetes Mellitus (BeDiaM) Study. Peroxisome proliferator-activated receptor-gamma2 polymorphism Pro12Ala is associated with nephropathy in type 2 diabetes:The Berlin Diabetes Mellitus (BeDiaM) Study. Diabetes. 2002;51(8):2653-7. 
59. Araki S, Koya D, Makiishi T, Sugimoto T, Isono M, Kikkawa R, et al. APOE polymorphism and the progression of diabetic nephropathy in Japanese subjects with type 2 diabetes: results of a prospective observational follow-up study. Diabetes Care. 2003;26(8):2416-20.

60. Limei L, Xiang K, Zheng T, Zhang R, Li M, Li J. Co-inheritance of specific genotypes of HSPG and ApoE gene increases risk of type 2 diabetic nephropathy. Mol Cell Biochem. 2003;254(1-2):353-8.

61. Caramori ML, Canani LH, Costa LA, Gross JL. The human peroxisome proliferator-activated receptor gamma2 (PPARgamma2) Pro12Ala polymorphism is associated with decreased risk of diabetic nephropathy in patients with type 2 diabetes. Diabetes. 2003;52(12):3010-3.

62. Canani LH, Capp C, Ng DP, Choo SG, Maia AL, Nabinger GB, et al. The fatty acid-binding protein-2 A54T polymorphism is associated with renal disease in patients with type 2 diabetes. Diabetes. 2005;54(11):3326-30.

63. Ilhan N, Kahraman N, Seçkin D, Ilhan N, Colak R. Apo E gene polymorphism on development of diabetic nephropathy. Cell Biochem Funct. 2007;25(5):527-32.

64. Kwon MK, Rhee SY, Chon S, Oh S, Woo JT, Kim SW, et al. Association between apoliprotein $\mathrm{E}$ genetic polymorphism and the development of diabetic nephropathy in type 2 diabetic patients. Diabetes Res Clin Pract. 2007;77(Suppl 1):S228-32.

65. Leiva E, Mujica V, Elematore I, Orrego R, Díaz G, Prieto M, et al. Relationship between apolipoprotein $E$ polymorphism and nephropathy in type-2 diabetic patients. Diabetes Res Clin Pract. 2007;78(2):196-201.

66. Erdogan M, Karadeniz M, Eroglu Z, Tezcanli B, Selvi N, Yilmaz C. The relationship of the peroxisome proliferator-activated receptor-gamma 2 exon 2 and exon 6 gene polymorphism in Turkish type 2 diabetic patients with and without nephropathy. Diabetes Res Clin Pract. 2007;78(3):355-9.

67. Pollex RL, Mamakeesick M, Zinman B, Harris SB, Hegele RA, Hanley AJ. Peroxisome proliferator-activated receptor gamma polymorphism Pro12Ala is associated with nephropathy in type 2 diabetes. J Diabetes Complications. 2007;21(3):166-71.

68. Georgopoulos A, Aras O, Tsai M. Codon-54 polymorphism of the fatty acid-binding protein 2 gene is associated with elevation of fasting and postprandial triglycerides in type 2 diabetes. J Clin Endocrinol Metab. 2000;85(9):3155-60.

69. Stumvoll $M$, Häring $H$. The peroxisome proliferators-activated receptor- $\gamma 2$ Pro12Ala polymorphism. Diabetes. 2002;51(8):2341-7. 